Page 1

\title{
Structures and Stabilities of Gold Oxide Films on Gold Surfaces in $\mathrm{O}_{2}$
}

\section{Atmosphere}

Keju Sun ${ }^{1,2^{*}}$, Masanori Kohyama ${ }^{2}$, Shingo Tanaka ${ }^{2}$ and Seiji Takeda ${ }^{1}$

1) The Institute of Scientific and Industrial Research, Osaka University, 8-1 Mihogaoka,

Ibaraki, Osaka 567-0047, Japan

2) Research Institute for Ubiquitous Energy Devices, National Institute of Advanced

Industrial Science and Technology (AIST), 1-8-31, Midorigaoka, Ikeda, Osaka 563-8577, Japan

*Corresponding author. Tel.: +81-72-751-9732; fax: +81-72-751-9714

Email: keju.sun@aist.go.jp 
Page 2

\begin{abstract}
The structures and stabilities of gold oxide films on gold surfaces in $\mathrm{O}_{2}$ atmosphere are investigated by a combination of thermodynamics and density functional theory (DFT) calculations. It is predicted that the stability of a gold oxide film on a gold surface depends on the formation energy of an isolated gold oxide film, the lattice mismatch, and the oxygen coverage. These predictions are subsequently confirmed by DFT calculations and thermodynamic analysis. The calculation results also display the dual chain structure is stable on most gold surfaces at low oxygen chemical potential except on a $\mathrm{Au}(111)$ surface, on which the graphene-like $12 \mathrm{MR}$ structure is more stable. While at the high oxygen chemical potential, the connected dual chain structure is the most stable structure because of the highest oxygen coverage and the lowest formation energy. The phase transformation point from the dual chain structure to the connected dual chain structure on a gold surface depends on the lattice mismatch between the connected dual chain and a gold surface.
\end{abstract}

\title{
Keywords:
}

Stabilities; Gold oxide film; Density functional theory calculations; Thermodynamic analysis 
Page 3

\section{Introduction}

Gold was considered as chemically inert for a very long time, for it is hard to be oxidized to $\mathrm{Au}_{2} \mathrm{O}_{3}[1,2,3]$. However, a gold oxide film stable under $500 \mathrm{~K}$ [4] can easily be produced on a gold surface, via adsorption of atomic oxygen [4,5], chemical oxidation [6,7], or photochemical oxidation with $\mathrm{O}_{3}[8,9]$. In past decades, numerous studies have been devoted to explore the nature of gold oxide films, since it is believed to be related to the unique oxidation catalysis of gold catalysts $[10,11]$. Despite the huge number of experimental and theoretical works, the structure of gold oxide films is still poorly understood. It even remains controversial whether the structure of atomic oxygen adsorption on gold is ordered or not, unlike sulfur or chlorine adsorption on $\mathrm{Au}(111)$, which induces a reconstruction to an ordered $\sqrt{ } 3 \times \sqrt{ } 3 \mathrm{R} 30^{\circ}$ pattern by low-energy electron diffraction (LEED) $[12,13]$. Some reports show that no ordered overlayer structure of atomic oxygen adsorption on $\mathrm{Au}(111)$ or $\mathrm{Au}(100)$ was observed by LEED $[4,7,8]$, while others show that the ordered structure can be formed by the same method or scanning tunneling microscopy (STM) $[14,15]$.

Recently, Uchiyama et al studied a real $\mathrm{Au} / \mathrm{CeO}_{2}$ catalyst in $\mathrm{O}_{2}$ atmosphere by in situ environmental transmission electron microscopy (ETEM) [16]. They found the gold surface is not steady with oxygen adsorption but fluctuates between $\mathrm{Au}(111), \mathrm{Au}(100)$ and $\mathrm{Au}(110)$ facets with time under the spatial resolution available on the environmental transmission electron microscope. The surface fluctuation makes it challenging to identify the structure of gold oxide films only by experimental methods. Theoretically, Shi and Stampfl investigated the structure and stability of atomic oxygen adsorption and surface oxide formation on $\mathrm{Au}(111)$ [17], $\mathrm{Au}(100)$ and $\mathrm{Au}(110)$ surfaces [18], and found a surface-oxide-like configuration, an added-atom (5x2)-O configuration, and a missing row (2x1)-2O configuration as the most stable structure on $\mathrm{Au}(111)$, 
Page 4

$\mathrm{Au}(100)$ and $\mathrm{Au}(110)$ surfaces, respectively. They suggested the $\mathrm{Au}(110)$ facets should be more stable at the higher oxygen chemical potentials. It should be noted that the oxygen saturated coverage in Shi and Stampfl's models with 0.3 ML on Au(111) and 0.1 ML on Au(100) is significantly lower than that in experimental conditions with about $0.8 \sim 1.2 \mathrm{ML}[4,7,8,19]$. So far as we know, the oxygen adsorption structures on gold surface are ambiguous at higher coverage.

Aiming to provide insights into the adsorption of oxygen on gold surfaces especially for the high oxygen coverage structures, we investigate the structures of surface gold oxide films on gold surfaces by the combination of thermodynamics and density functional theory (DFT) calculations $[18,20]$. In this work, we first propose several possible stable structures of gold oxide films based on the stable linear O-Au-O structure in our previous work [21]. Then stability of these gold oxide films on flat $\mathrm{Au}(111), \mathrm{Au}(100), \mathrm{Au}(110)$ surfaces and stepped $\mathrm{Au}(221)$ and $\mathrm{Au}(211)$ surfaces are calculated by DFT calculations, and the corresponding surface energies as a function of oxygen chemical potential are plotted in the phase diagram based on the thermodynamical method. Finally, the reason of fluctuation of gold surfaces in $\mathrm{O}_{2}$ atmosphere is discussed from the view of thermodynamics and dynamics of gold oxide films on gold surfaces.

\section{Methods}

Periodic DFT calculations using Vienna Ab Initio Simulation Package (VASP)[22] were performed to examine the structure and stability of gold oxide films on gold surfaces. The exchange-correlation energy and potential are described by generalized gradient approximation (GGA) in the form of Perdew-Burke-Ernzerhof (PBE) [23]. Plane waves are used to expand wave functions with a cutoff of $400 \mathrm{eV}$ for projector augmented wave (PAW) potentials.[24,25] 
The cutoff of $400 \mathrm{eV}$ is a reasonable compromise between the calculation accuracy and calculation speed based on the testing results (See supporting information in Figure 1S). The criteria for the convergence in the structural determination is the residual force less than $0.02 \mathrm{eV}$ $\AA^{-1}$. The calculated lattice constant for bulk $\mathrm{Au}$ was $4.17 \AA$, which is in reasonable agreement with the experimental value of $4.08 \AA$ and the previous theoretical value of $4.18 \AA$.[26] All surfaces including $\mathrm{Au}(111), \mathrm{Au}(100), \mathrm{Au}(110), \mathrm{Au}(221)$ and $\mathrm{Au}(211)$ surfaces with oxygen adsorption are modeled by a seven-layer slab except for the structures of Au surfaces covered by the graphene-like $12 \mathrm{MR}$ structures. For latter, the structures are modeled by a five-layer slab due to their large super cells. The adsorbed oxygen species on gold surfaces are placed on both sides and the vacuum thickness is $15 \AA$. Spin polarizations are considered and all the atoms in the slab are relaxed during all the calculations, and the high spin species is only found for the isolated $\mathrm{AuO}_{4}$ structure with five unpaired electrons. The transition state of gold atom diffusion on a $\mathrm{Au}(111)$ surface is searched by force reversed method [27].

It was reported that the low Miller indexed $\mathrm{Au}(111), \mathrm{Au}(100)$ and $\mathrm{Au}(110)$ surfaces are reconstructed to $\mathrm{Au}(111)-(23 * \sqrt{3}), \mathrm{Au}(100)-(1 \times 5)$ and $\mathrm{Au}(110)-(1 \mathrm{x} 2)$ surfaces in most cases [28]. However, in this work the simple unreconstructed surfaces instead of the reconstructed surfaces are calculated due to the following reasons: (1) The structures of unreconstructed surfaces are more simple than those of the reconstructed surfaces, therefore they are generally adopted in the theoretical studies [29]. (2) The recent TEM experimental results showed that the structures of $\mathrm{Au}(100)$ and $\mathrm{Au}(111)$ surfaces on gold cluster are unreconstructed in the vacuum [30], which indicates that the unreconstructed surfaces are stable especially on the gold nanoparticles.

To overcome the underestimation of weak cohesive energy of gold originated from $\mathrm{d}^{10}-\mathrm{d}^{10}$ interaction [31,32], the method of DFT with D3 correction [33] is used for all atoms in the 
Page 6

systems. The calculated surface energies of clean $\mathrm{Au}(111)$ surface, $\mathrm{Au}(100)$ and $\mathrm{Au}(110)$ surface without D3 correction are 46.4, 54.0 and $58.8 \mathrm{meV} / \AA^{2}$, respectively, which are in good agreement with the previous calculation results by DFT method of $46.2,53.1$ and $56.2 \mathrm{meV} / \AA^{2}$, correspondingly.[29] However, the calculated surface energies are significantly underestimated compared to the experimental value of $93.6 \mathrm{meV} / \AA^{2}$. [34] With D3 correction, the calculated surface energies are 89.6, 96.9 and $98.2 \mathrm{meV} / \AA^{2}$, respectively, which agree well with the experimental results.

The surface free energy of a gold surface with oxygen adsorption (O/Au system) is a measure to judge the stability. In this work, the surface free energy of an O/Au system is defined as

$$
\gamma(T, p)=\left(G_{O / A u}^{s l a b}-N_{A u} \mu_{A u}-N_{O} \mu_{O}\right) / A
$$

where $G_{O / A u}{ }^{s l a b}$ is the Gibbs free energy of the $\mathrm{O} / \mathrm{Au}$ system under consideration. $N_{A u}$ and $N_{O}$ are the numbers of $\mathrm{Au}$ and $\mathrm{O}$ atoms in the $\mathrm{O} / \mathrm{Au}$ system. $\mu_{A u}$ and $\mu_{O}$ are the chemical potentials of $\mathrm{Au}$ and $\mathrm{O}$ atoms and $A$ is the surface area of the $\mathrm{O} / \mathrm{Au}$ system. $\mu_{A u}=E_{A u}{ }^{\text {bulk }}$ and $G_{O / A u^{s l a b}}=E_{O / A u}{ }^{\text {slab }}$ are adopted since the contribution from $\mathrm{pV}$ term of solids in Gibbs free energy can be neglected for the pressure lower than $100 \mathrm{~atm}$ [17]. $\mu_{O}$ is normally defined as [20]

$$
\mu_{O}(T, p)=\frac{1}{2}\left[E_{O 2}+\Delta \mu_{O 2}\left(T, p^{0}\right)+k_{B} T \ln \left(\frac{p_{O 2}}{p^{0}}\right)\right]=\frac{1}{2}\left[E_{O 2}+\Delta \mu_{O 2}(T, p)\right]
$$

where $E_{O 2}$ is the total energy of the $\mathrm{O}_{2}$ molecule, $\Delta \mu_{O 2}\left(T, p^{0}\right)$ and $\Delta \mu_{O 2}(T, p)$ include the contribution from translations, rotations, vibrations of the $\mathrm{O}_{2}$ molecule at the pressure of $p^{0}(1$ atm) and $p$, respectively, which can be calculated by thermodynamics formulas or obtained from experimental thermodynamic tables. The DFT calculation with GGA exchange correlation functional overestimates the binding energy of $\mathrm{O}_{2}$ molecule in triplet state, which will induce an overestimation of all the surface energy of the surfaces with oxygen adsorption. Therefore, the 
argument of the oxygen chemical potential $\left(\Delta \mu_{O}=\Delta \mu_{O 2}(T, p) / 2\right)$ is used instead of two arguments of the temperature $(T)$ and the pressure $(p)$ in order to simplify the discussion. It should be noted that low $\Delta \mu_{O}$ corresponds to high temperature and low $\mathrm{O}_{2}$ pressure.

\section{Preliminary and general analysis}

The possible configurations of adsorbed oxygen on a gold surface are so numerous that it is hard to investigate all the possibilities. A preliminary screening is necessary based on some basic rules or principles before DFT calculations. According to our previous study [21,35], the $\pi$ bond plays a key role in the interaction between $\mathrm{Au}$ and $\mathrm{O}$ and the adsorbed atomic oxygen on gold thermodynamically prefers to form the linear O-Au-O structure. Likewise, it should be energetically favorable to form the crossed $\mathrm{AuO}_{4}$ structure consisting of two linear O-Au-O structures with strengthened Au-O $\pi$ bonding. Therefore, we believe that the surface gold oxide films should be composed of the linear O-Au-O structure (Figure 1a) and/or the crossed $\mathrm{AuO}_{4}$ structure (Figure 1b), since the linear O-Au-O structure could strengthen the Au-O interaction compared to those without linear O-Au-O structure [36]. Since the $\pi$ bonding is dominant for $\mathrm{Au}-\mathrm{O}$ interaction, a large flat structure with delocalized $\pi$ bonding in a wide range is expected. Furthermore, the fundamental to form a delocalized conjugated $\pi$ bond is the coplanar configuration of atoms, so the surface gold oxide film with one atomic layer is expected, which agrees well with the experimental results that only the surface top layer is oxidized [16] and the saturated oxygen coverage is about $1 \mathrm{ML}[19,37,38]$.

It was proven that the gold oxide with four-coordinated oxygen via $\mathrm{sp}^{3}$ hybridization is unstable compared to that with two-coordinated and three-coordinated oxygen by Shi and Stampfl [39]. Our DFT calculations also indicate that the four-coordinated oxygen is not as 
stable as three-coordinated and two-coordinated oxygen in $\mathrm{O} / \mathrm{Au}$ systems. This can be well understood since the contribution of $\pi$ bonding plays a key role in $\mathrm{O} / \mathrm{Au}$ systems [21]. The threecoordinated or two-coordinated oxygen with $\mathrm{sp}^{2}$ hybridization could form $\mathrm{Au}-\mathrm{O} \pi$ bonding via the non-hybridized $p$ orbital of oxygen and some $d$ orbitals of gold, while the four-coordinated oxygen with $\mathrm{sp}^{3}$ hybridization could not form Au-O $\pi$ bonding because all the orbitals of oxygen form $\sigma$ bonds. Therefore, the coordination number of oxygen in an $\mathrm{O} / \mathrm{Au}$ system could be two or three. In addition, the single coordinated oxygen is not considered as stable as two or three coordinated oxygen, since it will break the bond network in a gold oxide film and therefore destroy the delocalized conjugated $\pi$ bond.

According to our previous study, [21] the total binding energy increases with the coordination number, and thus it is easily predicted that the surface gold oxide with the highest coordination number for gold and oxygen will be the most stable structure. As we discussed above, the highest coordination numbers for gold and oxygen are four and three, so the most stable surface gold oxide should be composed of four-coordinated $\mathrm{Au}$ and three-coordinated $\mathrm{O}$, and the basic structure for gold is the crossed $\mathrm{AuO}_{4}$. It can be seen that the structure of bulk $\mathrm{Au}_{2} \mathrm{O}_{3}$ also obeys this rule $[39,40]$, where local configurations can be regarded as the crossed $\mathrm{AuO}_{4}$ structure and the maximum coordination number for oxygen is three.

Compared to an isolated gold film, the stability of the gold oxide film on a gold surface is more complicated, where the adhesion energy between the gold oxide film and a gold surface is also important. It is commonly believed that the adhesion energy is related to the lattice mismatch [41] between the gold oxide film and gold surface. The lattice constant includes two factors: the lattice side length $(u$ and $v)$, and the angle $(\alpha)$ between the sides. It is very convenient to judge the matching degree of angle. For example, the angle for the hexagonal structure with 
$60^{\circ}$ or $120^{\circ}$ is not equal to that of $90^{\circ}$ in a tetragonal structure, leading to a mismatch with each other from the angle. Certainly, the hexagonal structure also can be regarded as the tetragonal structure after the lattice constant multiplied by $\sqrt{ } 3$. There are several different methods to calculate the lattice mismatch degree $(\delta)$ between the film and surface, and in this work it is defined as [42],

$$
\delta=\frac{\left|L_{\text {Film }}-L_{\text {surface }}\right|}{L_{\text {surface }}} \times 100 \%
$$

where $L_{\text {Film }}$ is the lattice constant of a gold oxide film on a gold surface and $L_{\text {surface }}$ is the lattice constant of the gold surface. We predict that the oxide film on a gold surface with less lattice mismatch will be more stable.

The intrinsic stability of a gold oxide film on a gold surface depends on the stability of the isolated gold oxide film and the lattice mismatch. At $\mathrm{O}_{2}$ atmosphere, the stability is also depends on the oxygen chemical potential in the thermodynamics. From equations (1) and (2), it is concluded that the surface energy $(\gamma)$ has linear relationship with $-\Delta \mu_{O}\left(N_{O} / A\right)$. Thus the surface with lower oxygen coverage $\left(N_{O} / A\right)$ is more stable for lower oxygen chemical potential $\Delta \mu_{\mathrm{O}}$ (more negative), while the surface with higher oxygen coverage is more stable for high oxygen chemical potential. In this work, the surface coverage of oxygen in monolayer (ML) is defined as the numbers of oxygen atoms in each unit cell. Theoretically, there are three factors, the formation energy of an isolated gold oxide film, the lattice mismatch and the oxygen coverage, affecting the stability of gold oxide films on gold surfaces at given $\mathrm{O}_{2}$ atmosphere, and so DFT calculations with thermodynamics are necessary to find out the most stable structure. 
Page 10

\section{Results and discussion}

\subsection{The stability of the isolated gold oxide films}

As mentioned above, the gold oxide films should be composed of the linear O-Au-O structure and/or the cross $\mathrm{AuO}_{4}$ structure, and the coordination numbers are two or four for gold and two or three for oxygen. Therefore, we build four models of surface gold oxide based on the coordination number, as shown in Figure 1c-f. The coordination numbers of gold and oxygen are all two in Figure 1c (single chain), four and two in Figure 1d (dual chain), two and three in Figure 1e (graphene-like $12 \mathrm{MR}$ ), respectively. It should be noted that the structure with fourcoordinated gold and three-coordinated oxygen will break the symmetry of the crossed $\mathrm{AuO}_{4}$ structure, and then decrease the stability. Therefore, two kinds of gold atoms with the coordination numbers of four and two are found in Figure 1f (connected dual chain). Figure 1 also shows the formation energy $\left(E_{\text {form }}\right)$, as defined in the following,

$$
E_{\text {form }}=\frac{E_{O / A u}-N_{A u} E_{A u}^{b u l k}-\frac{N_{O}}{2} E_{O 2}}{N_{O}}
$$

where $E_{O / A u}, E_{A u}^{\text {bulk }}$, and $E_{O 2}$ are the total energies of the O/Au system, a bulk gold atom, and an $\mathrm{O}_{2}$ molecule, respectively. It is found that the formation energy decreased with the increase of the coordination number of gold or oxygen and the formation energy is the lowest for the connected dual chain. These results confirm our previous prediction that the surface gold oxide with the highest coordination number for gold and oxygen will be the most stable isolated structure. 
Page 11

\subsection{The stability of gold oxide films on $\mathrm{Au}(111)$ surface}

Having studied the stability of isolated surface gold oxide films, we then investigate the stability of gold oxide films on Au surfaces. The close-packed Au(111) surface is first investigated. Figure $2 \mathrm{a}$ and $2 \mathrm{~b}$ show the structures of single chain and dual chain on the $\mathrm{Au}(111)$ surface. Since the surface energy is related to the oxygen coverage, the structures of single chain and dual chain with the coverage of $1 / 2 \mathrm{ML}, 1 / 3 \mathrm{ML}$ and $1 / 4 \mathrm{ML}$ are calculated, where the coverage is set by changing the distance between the chains arrayed along the $\langle 011\rangle$ direction on the surface. Note that the density of the dual chains is always half of that of the single chains for the same coverage, due to the double density of oxygen atoms per chain (see Figure 2a and Figure $2 b$ for the coverage of 1/2 ML). In Figure $2 \mathrm{c}$, the dual chain (D) structure on $\mathrm{Au}(111)$ is always more stable than the single chain $(\mathrm{S})$ structure for each coverage. The reason can be assigned to the lower formation energy (per oxygen atom) of the isolated dual chain structure, $0.67 \mathrm{eV}$, in contrast to $1.24 \mathrm{eV}$ for the single chain structure (Figure $1 \mathrm{~d}$ and 1c). ). Since the sizes of the periodicity in the two kinds of chains are very similar, the lattice mismatch between the chain and all gold surfaces is similar for the single chain and dual chain. The influence of the oxygen chemical potential can also be ignored if the oxygen coverage is the same. As a result, the relative stability of isolated single chain and dual chain structures should be dominant, and the dual chain structure should be more stable than the single chain structure on all gold surfaces, thus only the dual chain structure is discussed thereafter.

The stability of graphene-like $12 \mathrm{MR}$ structures on $\mathrm{Au}(111)$ is also examined as shown in Figure 3a-d. Since both of the symmetry of the graphene-like 12 MR structure and the Au(111) surface are hexagonal, two structures with high symmetry can be formed for graphene-like 12 MR adsorption on the $\mathrm{Au}(111)$ surface. In one structure, the $\mathrm{Au}-\mathrm{O}$ bond in graphene-like $12 \mathrm{MR}$ 
is parallel to the $\mathrm{Au}-\mathrm{Au}$ bond in the $\mathrm{Au}(111)$ surface (Figure 3d), as denoted by eclipsed conformer. In the other one, the graphene-like $12 \mathrm{MR}$ film is rotated by $30^{\circ}$ degree (Figure 3a) compared to the eclipsed conformer, and it is called staggered conformer. As denoted in Figure 3a,d, proper two-dimensional supercells are naturally selected. Nine rings of graphene-like 12 MR and $49 \mathrm{Au}$ atoms constitute one coincidence supercell for the staggered conformer, and three rings and $16 \mathrm{Au}$ atoms constitute that for the eclipsed conformer. There is no substantial deformation in the gold oxide layer to attain such coincidence.

It is well known that the staggered conformer of ethane molecule has higher stability than the eclipsed conformer due to the strong repulsion between two groups of $\mathrm{CH}_{3} \sigma$ bonds for the eclipsed conformer (Figure 4). Likewise, the graphene-like $12 \mathrm{MR}$ on $\mathrm{Au}(111)$ with the staggered conformer should be more stable than those with the eclipsed conformer (Figure 4). The lattice mismatch degree for the staggered conformer is $133 \%$, which is almost equal to that for the eclipsed conformer by $131 \%$. Therefore the influence of lattice mismatch can be ignored when their relative stabilities are studied. In addition, the influence of oxygen chemical potential can also be ignored since the oxygen coverage of the staggered conformer with $0.367 \mathrm{ML}$ is almost same as the eclipsed conformer with 0.375 ML. As we can see in Figure 3e, the staggered conformer is more stable than the eclipsed conformer. The energy difference between the staggered and eclipsed conformers is about $0.32 \mathrm{eV}$ for each unit of graphene-like $12 \mathrm{MR}$, which may make only the staggered conformer to be observed. Since the repulsion between two groups with $\sigma$ bonds is general, this behavior could be used to explain the stability of similar structures, such as graphene on transition metals. It was reported that only the staggered structures were observed in the experiments.[43,44] 
Page 13

The work of Shi and Stampfl showed that the structure of graphene-like $12 \mathrm{MR}$ with some oxygen vacancies (Figure 3c) is more stable. [17] We thus investigate the stability of the graphene-like $12 \mathrm{MR}$ with oxygen vacancies on the $\mathrm{Au}(111)$ surface. For the eclipsed conformer, the graphene-like $12 \mathrm{MR}$ with oxygen vacancies (Figure 3c) is really more stable than that without oxygen vacancies (Figure 3d), which agrees well with Shi and Stampfl's results. However, for the staggered conformer, the graphene-like $12 \mathrm{MR}$ without oxygen vacancies is more stable. The role of oxygen vacancies in stabilizing the eclipsed conformer of graphene-like $12 \mathrm{MR}$ may be reducing the repulsive interactions between parallel $\mathrm{Au}-\mathrm{O} \sigma$ and $\mathrm{Au}-\mathrm{Au} \sigma$ bonds in the gold oxide film and the $\mathrm{Au}(111)$ surface, respectively. No matter the presence of oxygen vacancies or not, the staggered conformers are always more stable than the eclipsed conformer, and the stability of the staggered conformers can be assigned to the weaker repulsion between $\mathrm{Au}-\mathrm{Au} \sigma$ bond and $\mathrm{O}-\mathrm{Au} \sigma$ bond similar to ethane molecule.

We then turn to the stability of the connected dual chain structure on the Au(111) surface. Two typical structures of the connected dual chain adsorption are examined. In the first structure, the two-coordinated gold $(\mathrm{Au}(2))$ is located much lower than the four-coordinated gold $(\mathrm{Au}(4))$ so as to attain higher oxygen coverage as shown in Figure 5a. In this structure, the angle of O$\mathrm{Au}(2)-\mathrm{O}$ is far less than $180^{\circ}$. In the second structure of 3/4 ML, the $\mathrm{Au}(2)$ atoms are located almost in the same plane with $\mathrm{Au}(4)$, and the angle of $\mathrm{O}-\mathrm{Au}(2)-\mathrm{O}$ is close to $180^{\circ}$ as shown in Figure 5b. In the connected dual chain structure, the angle of $\mathrm{O}-\mathrm{Au}(2)-\mathrm{O}$, the lattice constant along the chain, the lattice mismatch degree and the oxygen coverage have mutual correlation as shown in Figure 5c. It is found that the surface energies of the connected dual chain structures are similar if the coverage is close to each other, since they have the similar formation energy and similar lattice mismatch degree. Around some coverage, there are several similar connected 
dual chain configurations, which make it possible to transform from one configuration to another. In our results, the flat connected dual chain structure with the coverage of $3 / 4 \mathrm{ML}$ is the most stable in a wider range of oxygen chemical potential, while it will transform to that with higher oxygen coverage (for instant, 7/9 ML) for higher oxygen chemical potential, since the structure with high oxygen coverage is more stable for high oxygen chemical potential.

We examined the relative stability among the single chain, dual chain, graphene-like $12 \mathrm{MR}$ and connected dual chain structures on the $\mathrm{Au}(111)$ surface as a function of the oxygen chemical potential. Except for the single chain structure which is always more unstable than the dual chain structure, the surface energies of other three structures are shown in Figure 6, where each line of the three structures reveals several segments with different slopes, corresponding to the most stable configuration with a difference coverage value for each region of the oxygen chemical potential, via the examination of various coverage configurations. In Figure 6 , it is found that the graphene-like $12 \mathrm{MR}$ structure is the most stable at lower oxygen chemical potential, whereas at higher oxygen chemical potential, the connected dual chain structure is the most stable, due to its higher oxygen coverage. Since the formation energy of the isolated graphene-like $12 \mathrm{MR}, 1.21$ $\mathrm{eV}$, is higher than those of dual chain structure and connected dual chain structures, $0.67 \mathrm{eV}$ and $0.45 \mathrm{eV}$, respectively, the stability of graphene-like $12 \mathrm{MR}$ on the $\mathrm{Au}(111)$ surface can be assigned to the high adhesive energy due to its hexagonal symmetry similar to the $\mathrm{Au}(111)$ surface.

\subsection{The stability of gold oxide films on $\mathrm{Au}(100), \mathrm{Au}(110), \mathrm{Au}(221)$ and $\mathrm{Au}(211)$ surfaces}

Similarly to the gold-oxide films on $\mathrm{Au}(111)$ surface, we examined the stability of the films on $\mathrm{Au}(100)$ surface for the dual chain, connected dual chain and graphene-like $12 \mathrm{MR}$ structures as shown in Figure 7. In Figure 7d, it is found that the dual chain structure is always more stable 
than the graphene-1ike $12 \mathrm{MR}$ structure on the $\mathrm{Au}(100)$ surface. The graphene-like $12 \mathrm{MR}$ structure is unstable on the $\mathrm{Au}(100)$ surface compared to that on the $\mathrm{Au}(111)$ surface, which can be ascribed to the larger lattice mismatch. The graphene-like $12 \mathrm{MR}$ has hexagonal symmetry similar to the $\mathrm{Au}(111)$ surface, which is quite different from the $\mathrm{Au}(100)$ surface with square symmetry.

When the oxygen chemical potential is low, the dual chain structure is more stable, and the connected dual chain structure becomes more stable at higher oxygen chemical potential. In Figures $7 \mathrm{a}$ and $7 \mathrm{~b}$, we can see that the dual chain structure and connected dual chain structure have similar configurations, and the lattice mismatch should be the same. The relative stability depending on the oxygen chemical potential can be explained by the oxygen coverage. The dual chain structure with lower oxygen coverage is more stable at the low oxygen chemical potential, while the connected dual chain structure with higher oxygen coverage is more stable at the high oxygen chemical potential. On the $\mathrm{Au}(100)$ surface, the phase transformation point from the dual chain structure to the connected dual chain structure is $-0.21 \mathrm{eV}$, which is lower than the point of the formation of $\mathrm{Au}_{2} \mathrm{O}_{3}(-0.14 \mathrm{eV})$.

Similar gold-oxide film adsorption was examined on the $\mathrm{Au}(110)$ surface for dual chain, connected dual chain and graphene-like 12 MR structures as shown in Figure 8. The graphenelike $12 \mathrm{MR}$ structure is also unstable on $\mathrm{Au}(110)$, since the symmetry of the $\mathrm{Au}(110)$ surface is tetragonal, differently from that of the graphene-like $12 \mathrm{MR}$ structure. The dual chain structure is the most stable in a very wide range of the oxygen chemical potential, which agrees well with Shi and Stampfl's result [18]. This can be explained by the lattice mismatch with the Au(110) surface. In Figures $8 \mathrm{a}$ and $8 \mathrm{~b}$, the lattice mismatch degrees are zero and $50 \%$ for the dual chain 
Page 16

and connected dual chain structures, respectively. Thus the phase transformation point from the dual chain structure to the connected dual chain structure is much higher (higher than $-0.14 \mathrm{eV}$ ).

From the above results, the graphene-like $12 \mathrm{MR}$ structure with hexagonal symmetry seems to be stable only on the $\mathrm{Au}(111)$ surface with similar hexagonal symmetry. Thus for the stepped $\mathrm{Au}(221)$ and $\mathrm{Au}(211)$ surfaces with tetragonal symmetry, only the dual chain and connected dual chain structures were examined. It is easily found that the dual chain structure matches well with the $\mathrm{Au}(221)$ surface in Figure 9a, while the lattice mismatch degree of the connected dual chain on the $\mathrm{Au}(221)$ surface is as high as $33 \%$ (Figure 9b). As shown in Figure 9c, the phase transformation point from the dual chain structure to the connected dual chain structure is higher than $-0.14 \mathrm{eV}$. On the $\mathrm{Au}(211)$ surface, the connected dual chain structure is reconstructed to a partly connected dual chain structure as shown in Figure 10b, due to high lattice mismatch. After reconstruction, the partly connected dual chain structure matches well with the $\mathrm{Au}(211)$ surface, and thus the phase transformation point from the dual chain structure to the connected dual chain structure $(-0.18 \mathrm{eV})$ is lower than $-0.14 \mathrm{eV}$.

On the comparison of the stability of the gold oxide films among various Au surfaces examined, it is found that the dual chain structure is always stable at the low oxygen chemical potential except for the $\mathrm{Au}(111)$ surface, due to the high match with the surfaces. For the connected dual chain structure, it only matches well with the $\mathrm{Au}(100)$ and $\mathrm{Au}(211)$ surfaces, and so the phase transformation point from the dual chain structure to the connected dual chain structure is lower than $-0.14 \mathrm{eV}$ on these gold surfaces. On the other hand, the phase transformation point is higher than $-0.14 \mathrm{eV}$ on the $\mathrm{Au}(110)$ and $\mathrm{Au}(221)$ surfaces, due to the large lattice mismatches for these surfaces. In this way, it can be said that the relative stability of gold oxide films on gold surfaces is strongly dependent on the lattice mismatch degree. 
According to our calculations, $\mathrm{Au}_{2} \mathrm{O}_{3}$ is the stable crystal phase, when the oxygen chemical potential is higher than $-0.14 \mathrm{eV}$. However, so far as we know, there is no report that $\mathrm{Au}_{2} \mathrm{O}_{3}$ was prepared by the method of gold direct oxidation. The oxidation of gold to $\mathrm{Au}_{2} \mathrm{O}_{3}$ may be hindered by the dynamic reason if the oxygen chemical potential is not high enough. Therefore the gold film is still possibly stable for the oxygen potential higher than $-0.14 \mathrm{eV}$. For the situation with very high oxygen chemical potential $(>0.1 \mathrm{eV}$, for instance), the connected dual chain structure is stable, since its oxygen coverage is highest in all the structures studied. Since the lattice constants of gold oxide films are different from the Au surfaces, we expect that the difference could be observed by high resolution transmission electron microscopy (HRTEM) directly in $\mathrm{O}_{2}$ atmosphere.

\subsection{The discussion on the fluctuation of gold surfaces in $\mathrm{O}_{2}$ atmosphere}

We are now in a position to address the observed surface fluctuation of Au clusters in $\mathrm{O}_{2}$ atmosphere. Table 1 shows the surface energies of clean gold surfaces, and those of oxidized gold surfaces at $\Delta \mu_{\mathrm{O}}=-0.14 \mathrm{eV}$ obtained as gold-oxide film adsorption in the present examination. It is found that all the gold surface energies are decreased from $\gamma 1$ to $\gamma 2$. According to Wulff construction [45], the distance from the center of a crystal to the surface is dependent on the surface energy. Therefore the variation of the surface area due to the $\mathrm{O}_{2}$ atmosphere is relative to the variation of the corresponding surface energy $(\Delta)$. From Table 1, the variation of the $\operatorname{Au}(111)$ surface energy is the smallest, and the $\mathrm{Au}(111)$ surface energy becomes relatively higher in the $\mathrm{O}_{2}$ atmosphere, implying that the area of the $\mathrm{Au}(111)$ surface should be dramatically decreased. The variation of the $\mathrm{Au}(110)$ surface energy is the largest, leading to the lowest value in the $\mathrm{O}_{2}$ atmosphere, which indicates the increase of the $\mathrm{Au}(110)$ surface area. The results agree well with Shi and stampfl's calculation results [18] and Uchiyama's experimental results that the $\mathrm{Au}(110)$ 
surfaces appear in $\mathrm{O}_{2}$ atmosphere [16]. Since the variation of the surface energy is dependent on the oxygen chemical potential, the shape of gold clusters and the structure of gold oxide films should change thermodynamically according to the oxygen chemical potential change.

To investigate the surface fluctuation of Au clusters from the viewpoint of dynamics, we calculated the gold-atom adsorption and diffusion on the $\mathrm{Au}(111)$ surface. It is found that the adsorption energies of a gold atom on the $\mathrm{Au}(111)$ surface are $-2.29,-2.91$, and $-2.86 \mathrm{eV}$ on the top, fcc and hcp sites, respectively, indicating that the interaction between a gold atom and a gold surface is strong, due to the strong $\mathrm{Au}-\mathrm{Au}$ metallic bond. Our calculation results on the $\mathrm{Au}(111)$ surface show that the diffusion barrier from the hcp site to the fcc site is $0.08 \mathrm{eV}$ and that from fcc to hcp is $0.13 \mathrm{eV}$. Although the adsorption of a gold atom on a gold surface is very strong (> $2 \mathrm{eV}$ ), the diffusion barrier is only $0.08 \sim 0.13 \mathrm{eV}$. The low diffusion barrier originates from the $\mathrm{Au}-\mathrm{Au}$ metallic bond characters such as relative softness and isotropic nature, typical of noble metals, different from transition metals or ionic or covalent materials. The barrier is so low that the diffusion of gold atoms on gold surfaces very easily occurs, which should support the formation of gold oxide films according to the $\mathrm{O}_{2}$ atmosphere. As a result, the surface fluctuation of $\mathrm{Au}$ clusters should easily occur in $\mathrm{O}_{2}$ atmosphere from the viewpoint of dynamics.

\section{Conclusions}

The stability of gold oxide films on various gold surfaces in $\mathrm{O}_{2}$ atmosphere is investigated. Based on the preliminary and general analysis, it is predicted that the O/Au system with the crossed $\mathrm{AuO}_{4}$ composed of three-coordinated oxygen and four-coordinated gold atoms is the most stable structure for an isolated gold oxide film. While for gold oxide films on gold surfaces in $\mathrm{O}_{2}$ atmosphere, the formation energy of the isolated gold oxide film, the lattice mismatch and 
the oxygen coverage affect the stability. The structure with less lattice mismatch should be more stable, and the structure with higher oxygen coverage should be more stable at high oxygen chemical potential. By thermodynamic analysis based on DFT calculations, it is found that the graphene-like $12 \mathrm{MR}$ structure is stable only on the Au(111) surface at low oxygen chemical potential, due to the symmetry match between the film and the surface. On all the other gold surfaces, the dual chain structure is the most stable at the low oxygen chemical potential, and the connected dual chain structure becomes more stable at the high oxygen chemical potential due to its higher oxygen coverage. The transformation point from the dual chain structure to the connected dual chain structure depends on the lattice mismatch between the connected dual chain structure and the gold surface. The surface fluctuation of Au clusters in $\mathrm{O}_{2}$ atmosphere can be assigned to the variation of the surface energies due to the generation of gold oxide films, according to the oxygen chemical potential change in thermodynamics, which should be assisted by low diffusion barriers for gold atoms on gold surfaces in dynamics.

Acknowledgment s

This study was supported by Grant-in-Aid for Specially Promoted Research Grant No. 19001005 from the Ministry of Education, Culture, Sports, Science and Technology of Japan (MEXT). A part of this study was supported by the Management Expenses Grants for National Universities Corporations from MEXT, and by Japan Science and Technology Agency (JST), Core Research for Evolutional Science and Technology (CREST). 

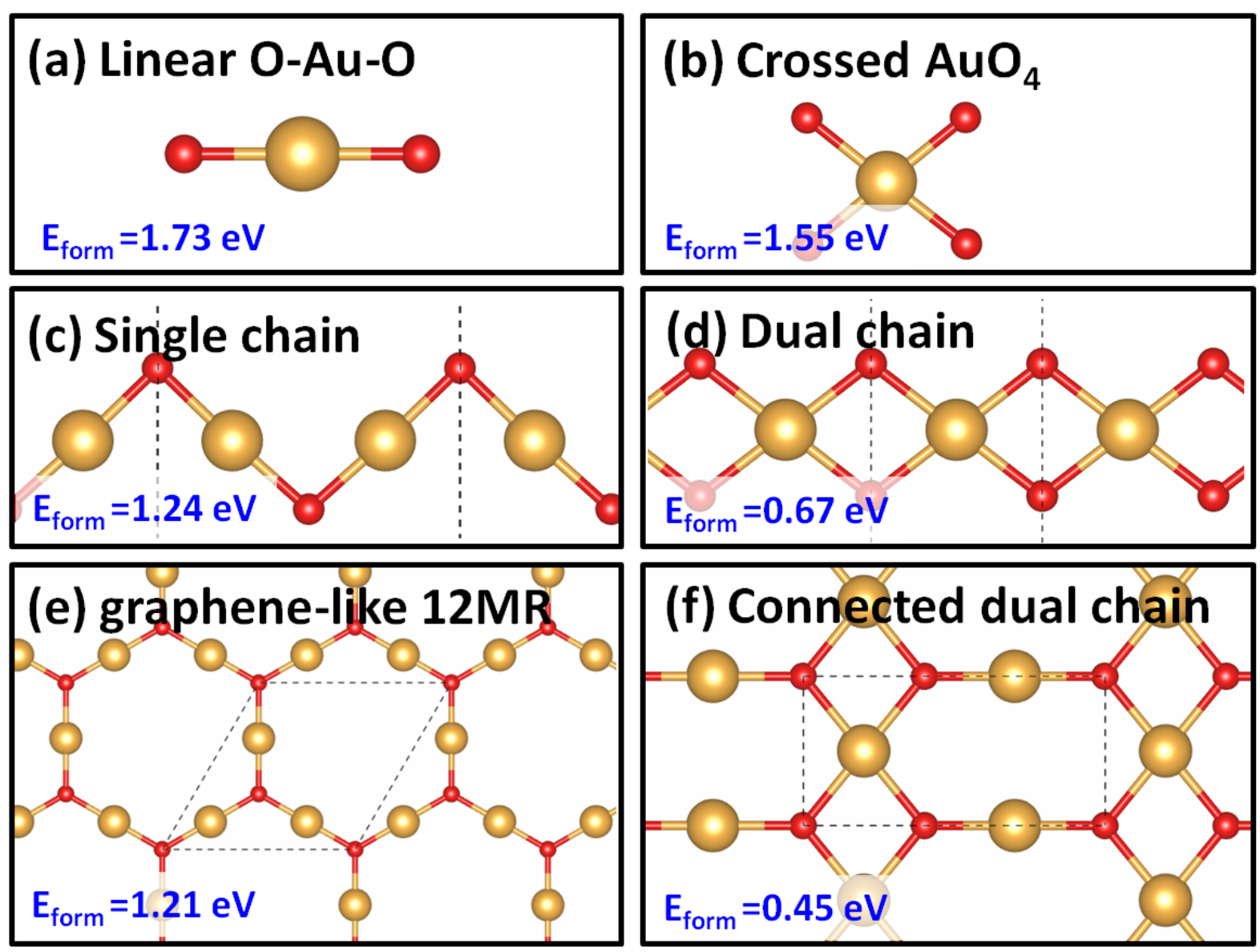

Figure 1. The proposed structures of isolated O/Au systems based on the coordination numbers of gold and oxygen. The gold balls denote the gold atoms and the red ones are oxygen atoms, and the dashed lines display the unit cell. (similarly hereinafter). 

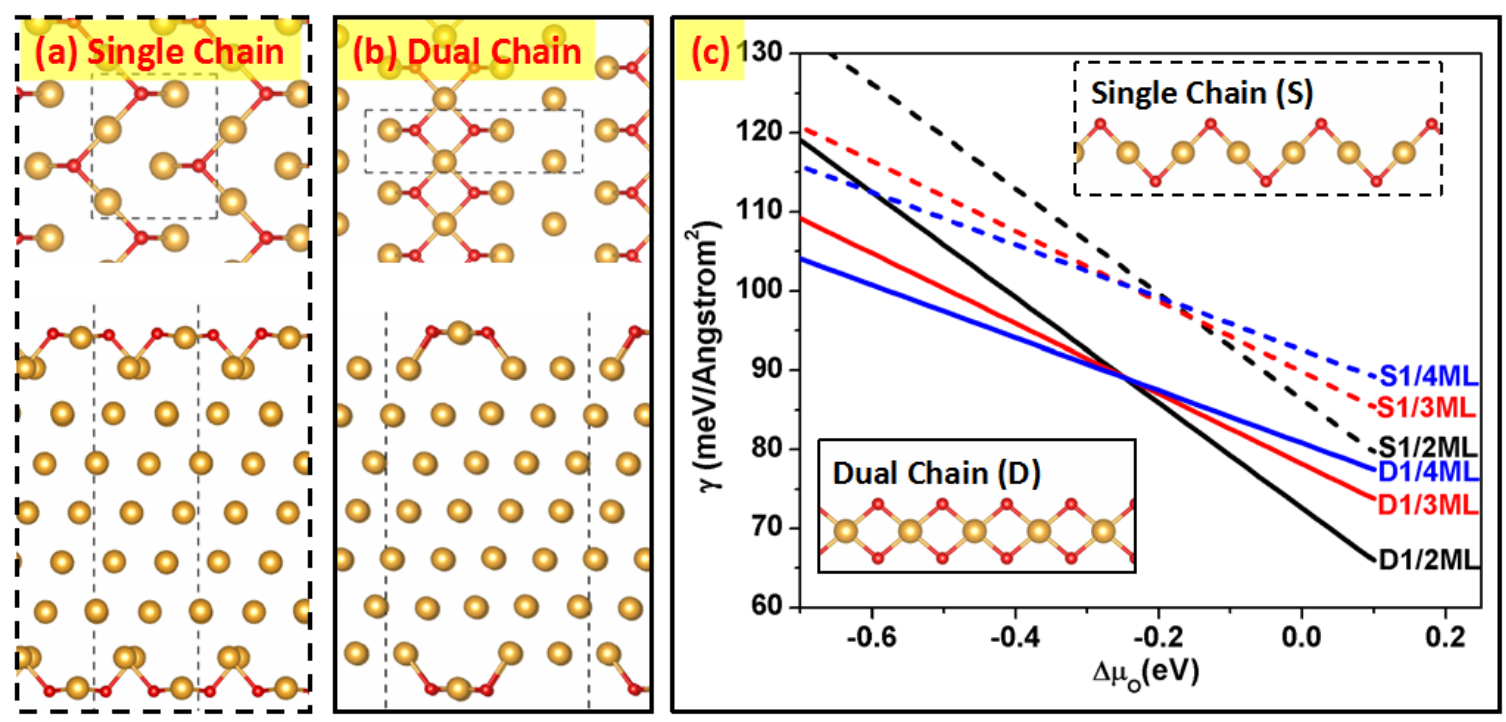

Figure 2. The structures of single chain (a) and dual chain (b) on the $\mathrm{Au}(111)$ surface and corresponding surface energies for these structures as a function of the oxygen chemical potential (c). In the figures a and b, the top parts show the top view and the bottom parts display the side view. To clarify, only the atoms in the top two layers are shown in the top view (similarly hereinafter). The labels "1/2 ML", "1/3 ML" and "1/4 ML" denote the oxygen coverage in the corresponding structures. 

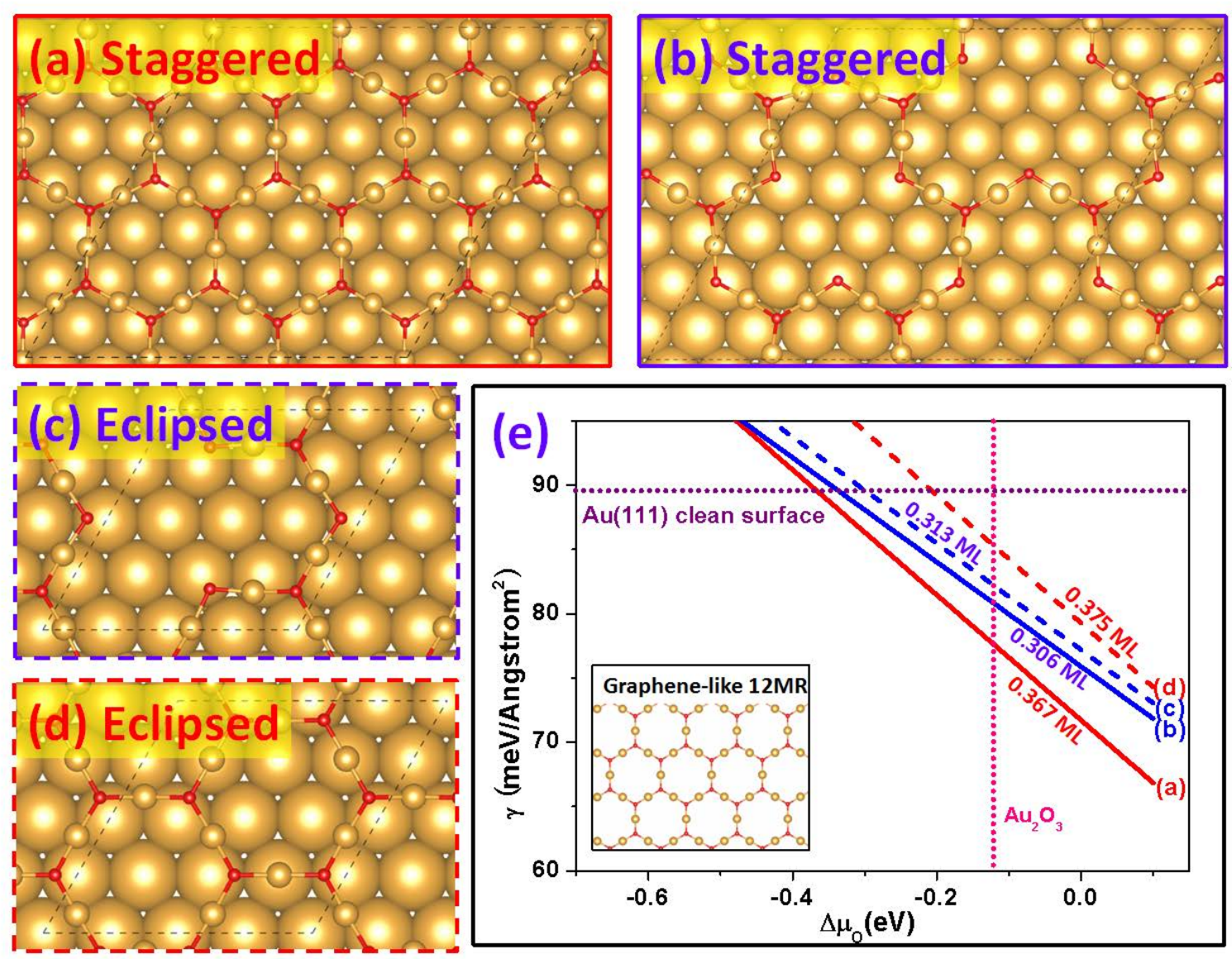

Figure 3. Top view of four different structures of graphene-like $12 \mathrm{MR}$ on the Au(111) surface (a-d) and corresponding surface energies for these structures as a function of the oxygen chemical potential (e). To clarify, the atoms in the top layer are marked as small balls and the atoms in the second layer are marked as large balls. The surface energy of a clean $\mathrm{Au}(111)$ surface is also shown as reference. 
Page 23

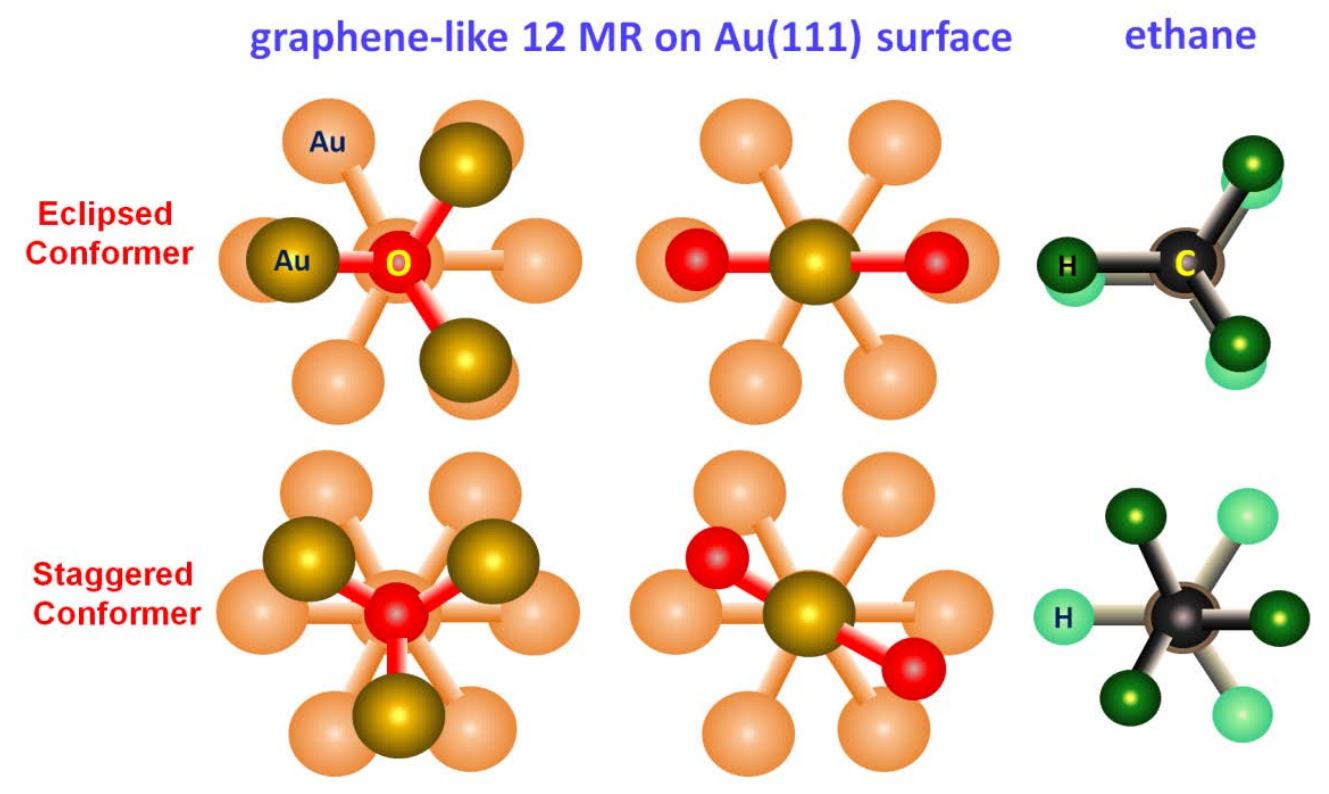

Figure 4. The top view of local configurations of graphene-like $12 \mathrm{MR}$ on the Au(111) surface and ethane molecules with eclipsed conformer and staggered conformer. The deep color balls show the atoms in the top layer and the light color balls denote the atoms in the second layer. 

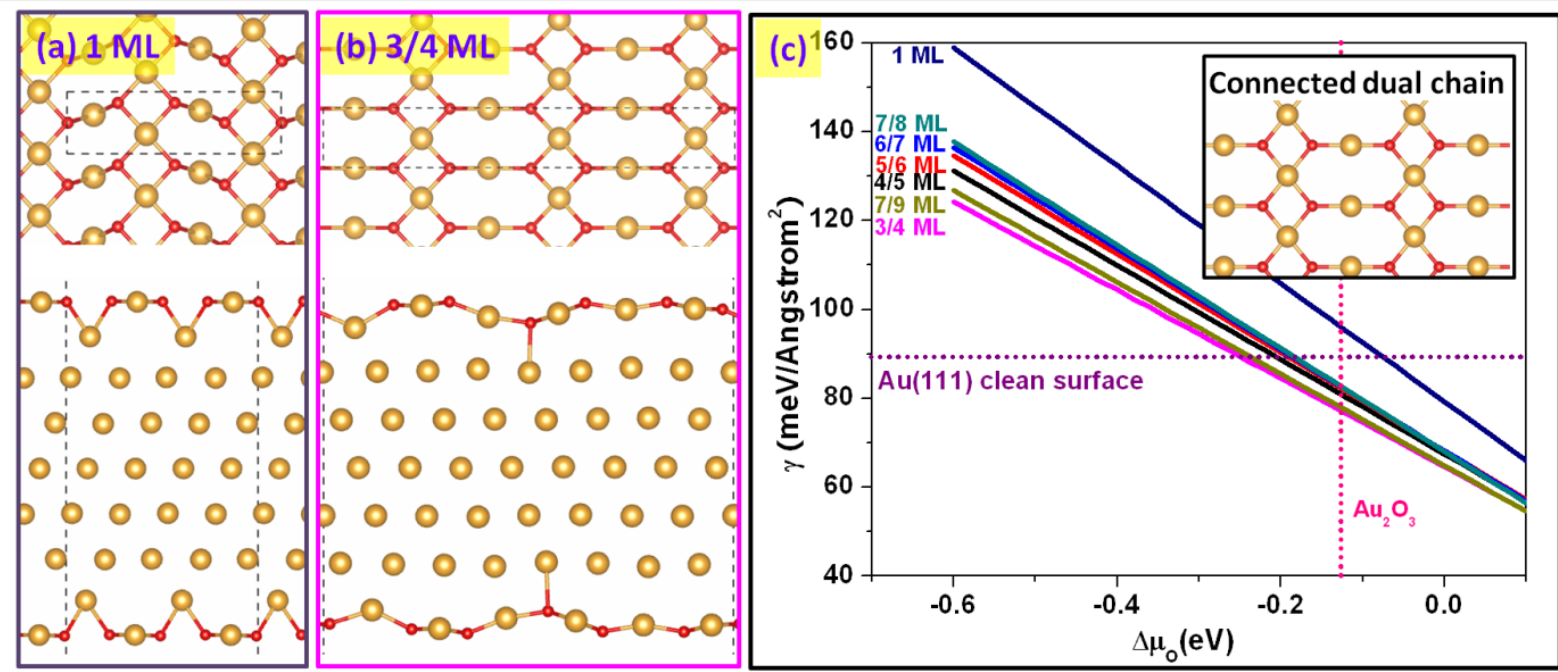

Figure 5. Two typical structures of connected dual chain on the $\mathrm{Au}(111)$ surface (a,b) and the surface energies for these two structures and some similar structures at difference coverage as a function of the oxygen chemical potential (c). The surface energy of a clean Au(111) surface is also shown as reference. 
Page 25

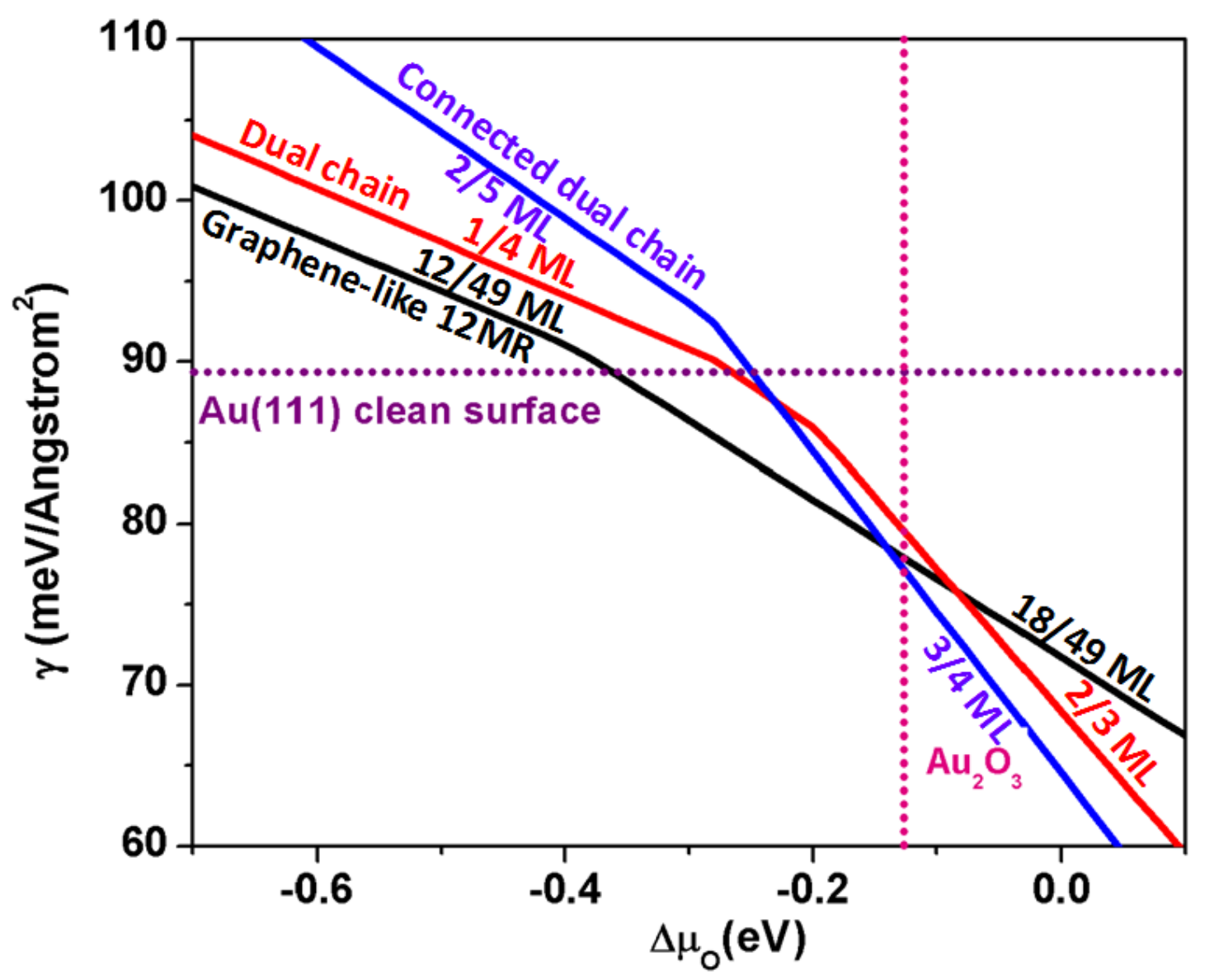

Figure 6. The surface energies for the structures of dual chain, connected dual chain and graphene-like $12 \mathrm{MR}$ on the $\mathrm{Au}(111)$ surface as a function of the oxygen chemical potential. The surface energy of a clean $\mathrm{Au}(111)$ surface is also shown as reference. Segments in each line with different slopes represent the most stable structure with different oxygen coverage for each structure in each range of the oxygen chemical potential. 
Page 26
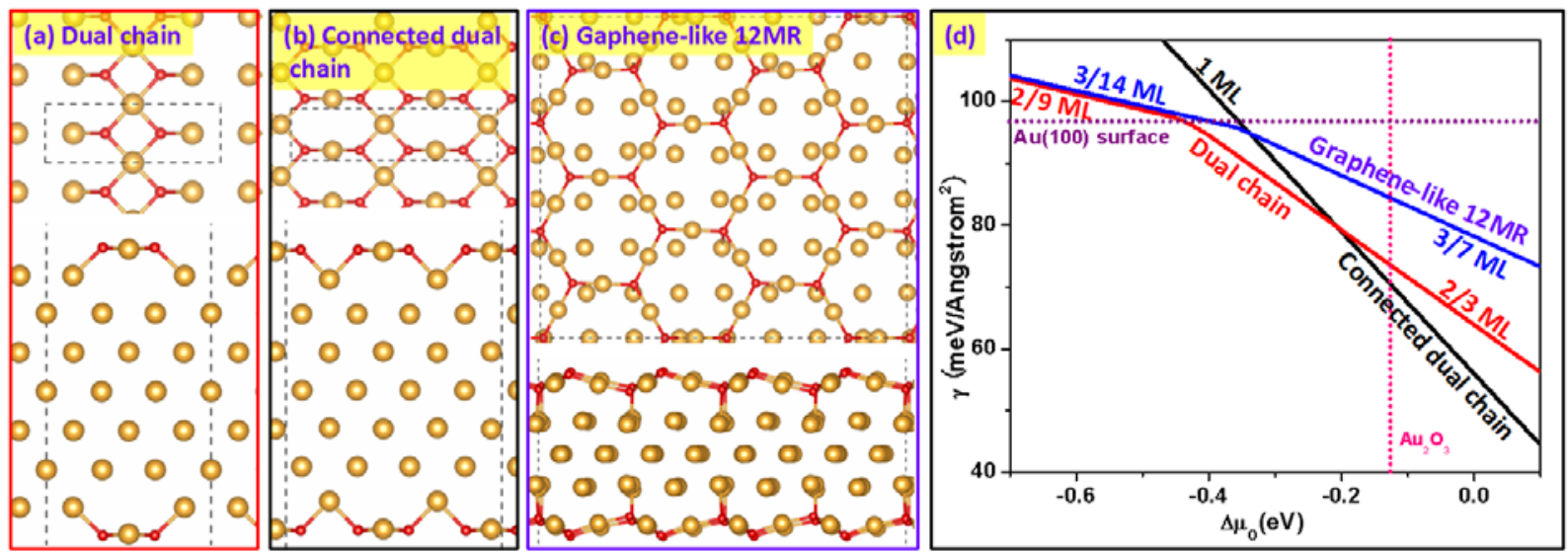

Figure 7. Three different structures of dual chain (a), connected dual chain (b) and graphene-like $12 \mathrm{MR}$ (c) on the $\mathrm{Au}(100)$ surface and corresponding surface energies as a function of the oxygen chemical potential (d). The surface energy of a clean $\mathrm{Au}(100)$ surface is also shown as reference. 
Page 27
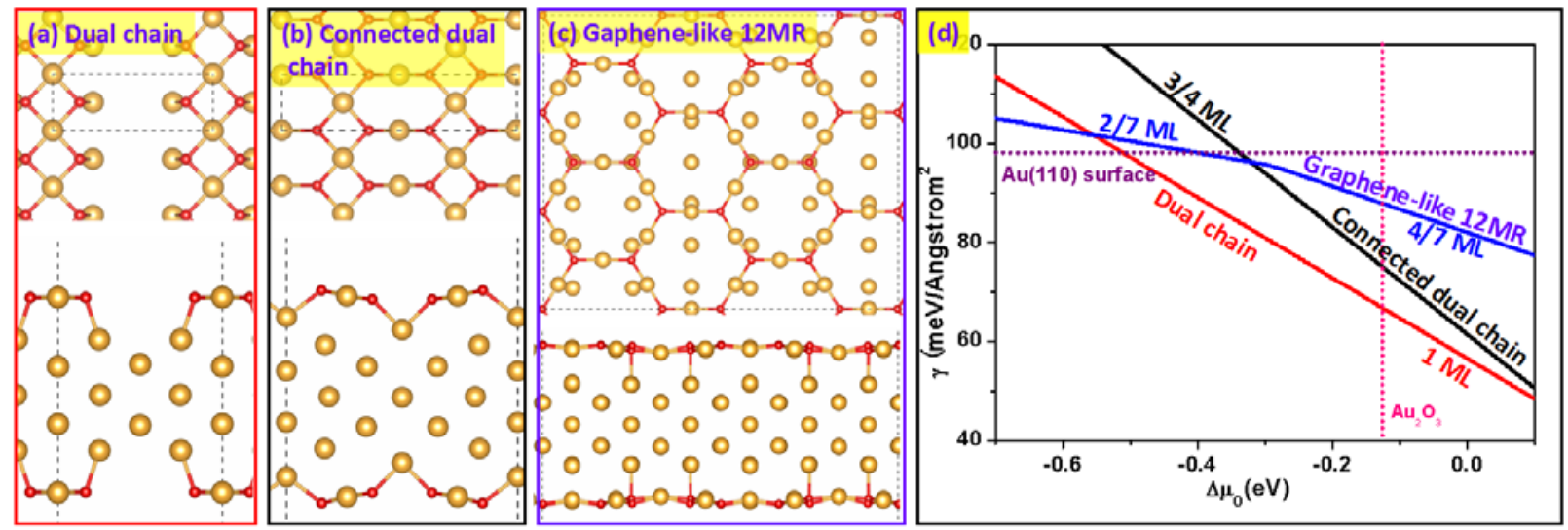

Figure 8. Three different structures of dual chain (a), connected dual chain (b) and graphene-like $12 \mathrm{MR}(\mathrm{c})$ on the $\mathrm{Au}(110)$ surface and corresponding surface energies for these structures as a function of the oxygen chemical potential (d). The surface energy of a clean $\mathrm{Au}(110)$ surface is also shown as reference. 
Page 28
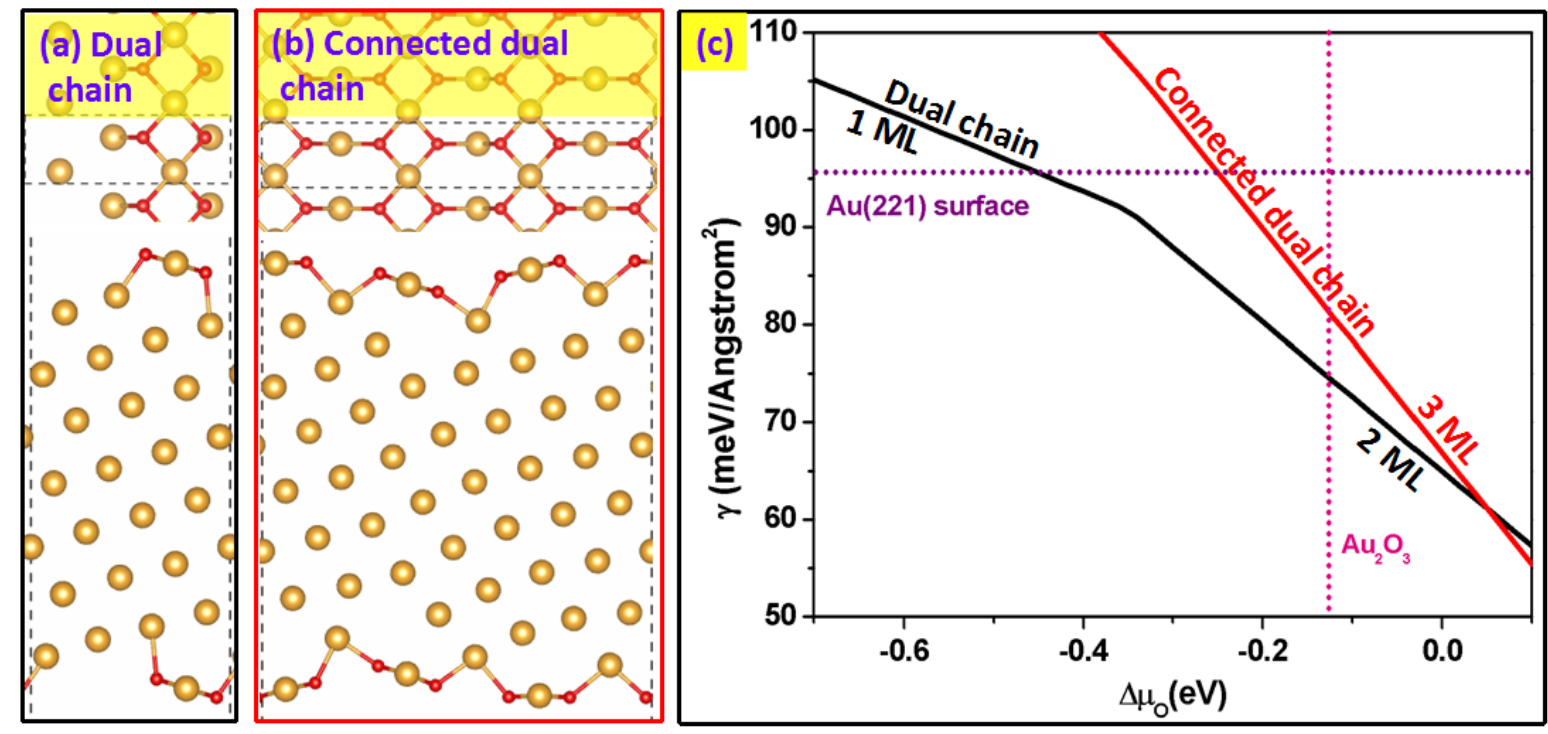

Figure 9. Two structures of dual chain (a), connected dual chain (b) on the $\mathrm{Au}(221)$ surface and corresponding surface energies for these structures as a function of the oxygen chemical potential (c). The surface energy of a clean $\mathrm{Au}(221)$ surface is also shown as reference. 
Page 29
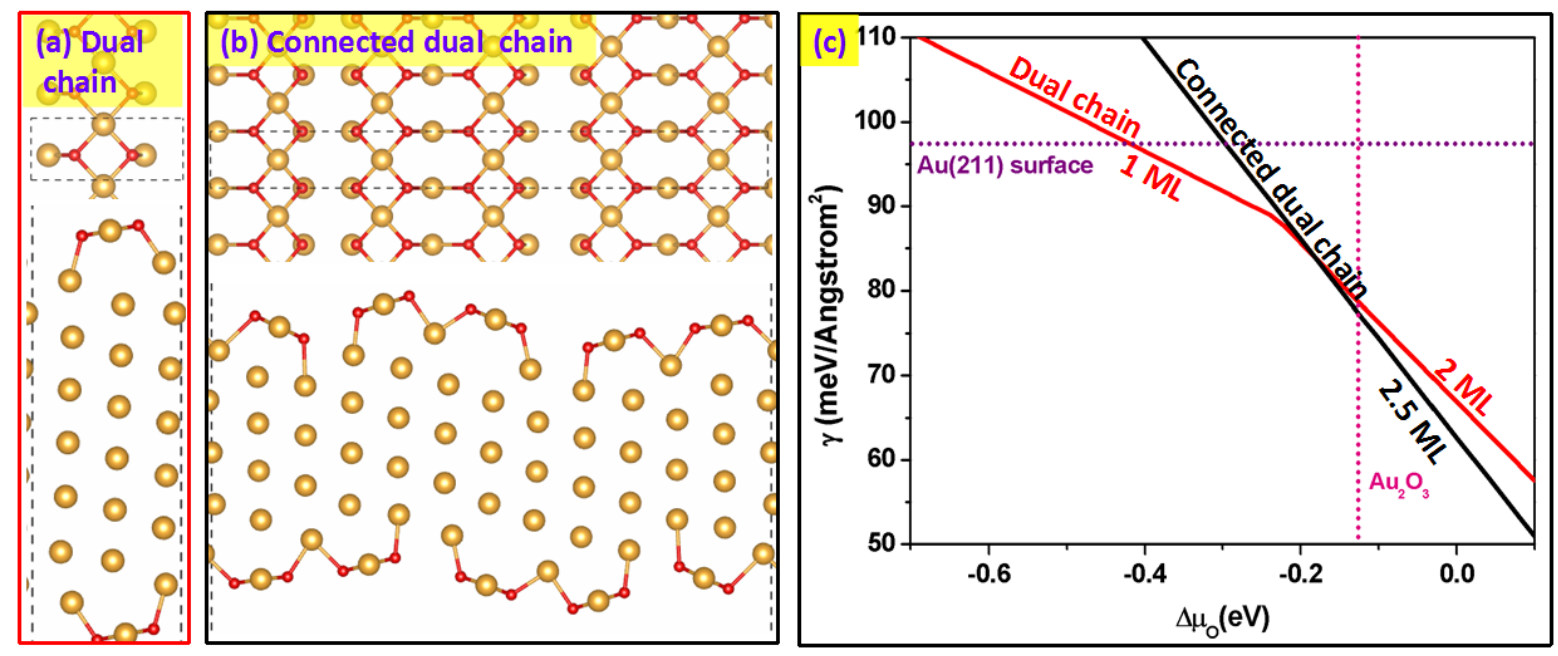

Figure 10. Two structures of dual chain (a) and connected dual chain (b) on the $\mathrm{Au}(211)$ surface and corresponding surface energies for these structures as a function of the oxygen chemical potential (c). The surface energy of a clean $\mathrm{Au}(211)$ surface is also shown as reference. 
Page 30

Table 1. The surface energies $\left(\mathrm{meV} / \AA^{2}\right)$ of a clean surface $(\gamma 1)$ and an oxidized surface at $\Delta \mu_{\mathrm{O}}=-0.14 \mathrm{eV}(\gamma 2)$ and the variation of the surface energy $(\Delta)$ from $\gamma 1$ to $\gamma 2$.

\begin{tabular}{cccc}
\hline & $\gamma 1$ (clean) & $\gamma$ 2(oxidized) & $\Delta=(1-\gamma 2 / \gamma 1)$ \\
\hline $\operatorname{Au}(111)$ & 89.6 & 78.5 & $12.4 \%$ \\
$\mathrm{Au}(100)$ & 96.9 & 72.0 & $25.7 \%$ \\
$\mathrm{Au}(110)$ & 98.2 & 67.9 & $30.9 \%$ \\
$\mathrm{Au}(221)$ & 95.1 & 75.7 & $20.4 \%$ \\
$\mathrm{Au}(211)$ & 97.1 & 79.1 & $18.5 \%$ \\
\hline
\end{tabular}

\section{Reference}

[1] R.H. Gerke, M.D. Rourke, J. Am. Chem. Soc. 49 (1927) 1855.

[2] T.E. Jones, S. Piccinin, C. Stampfl, Mater. Chem. Phys. 141 (2013) 14.

[3] J. Szlachetko, J. Sa, M. Nachtegaal, U. Hartfelder, J.C. Dousse, J. Hoszowska, D.L.A.

Fernandes, H.Q. Shi, C. Stampfl, J. Phys. Chem. Lett. 5 (2014) 80.

[4] K.A. Davis, D.W. Goodman, J. Phys. Chem. B 104 (2000) 8557.

[5] J.M. Gottfried, K.J. Schmidt, S.L.M. Schroeder, K. Christmann, Surf. Sci. 511 (2002) 65.

[6] H. Ron, I. Rubinstein, Langmuir 10 (1994) 4566.

[7] D.H. Parker, B.E. Koel, J. Vac. Sci. Technol., A 8 (1990) 2585.

[8] N. Saliba, D.H. Parker, B.E. Koel, Surf. Sci. 410 (1998) 270.

[9] J.A.M. Sondaghuethorst, L.G.J. Fokkink, Langmuir 8 (1992) 2560.

[10] N.D.S. Canning, D. Outka, R.J. Madix, Surf. Sci. 141 (1984) 240.

[11] D.A. Outka, R.J. Madix, Surf. Sci. 179 (1987) 351.

[12] D.C. Jackson, A. Chaudhuri, T.J. Lerotholi, D.P. Woodruff, R.G. Jones, V.R. Dhanak, Surf. Sci. 603 (2009) 807.

[13] G.N. Kastanas, B.E. Koel, Appl. Surf. Sci. 64 (1993) 235.

[14] B.K. Min, A.R. Alemozafar, D. Pinnaduwage, X. Deng, C.M. Friend, J. Phys. Chem. B 110 (2006) 19833.

[15] J. Chevrier, L. Huang, P. Zeppenfeld, G. Comsa, Surf. Sci. 355 (1996) 1.

[16] T. Uchiyama, H. Yoshida, Y. Kuwauchi, S. Ichikawa, S. Shimada, M. Haruta, S. Takeda, Angew. Chem. Int. Ed. 50 (2011) 10157.

[17] H. Shi, C. Stampfl, Phys. Rev. B 76 (2007) 075327. 
[18] H.Q. Shi, C. Stampfl, Phys. Rev. B 77 (2008) 094127.

[19] T. Fukushima, S. Galvagno, G. Parravano, J. Catal. 57 (1979) 177.

[20] W.X. Li, C. Stampfl, M. Scheffler, Phys. Rev. Lett. 90 (2003) 256102.

[21] K.J. Sun, M. Kohyama, S. Tanaka, S. Takeda, J. Phys. Chem. A 116 (2012) 9568.

[22] G. Kresse, J. Hafner, Phys. Rev. B 48 (1993) 13115.

[23] J.P. Perdew, K. Burke, M. Ernzerhof, Phys. Rev. Lett. 77 (1996) 3865.

[24] P.E. Blochl, Phys. Rev. B 50 (1994) 17953.

[25] G. Kresse, D. Joubert, Phys. Rev. B 59 (1999) 1758.

[26] M. Mavrikakis, P. Stoltze, J.K. Norskov, Catal. Lett. 64 (2000) 101.

[27] K.J. Sun, Y.H. Zhao, H.Y. Su, W.X. Li, Theor. Chem. Acc. 131 (2012) 1118.

[28] R. Meyer, C. Lemire, S.K. Shaikhutdinov, H. Freund, Gold Bulletin 37 (2004) 72.

[29] N.E. Singh-Miller, N. Marzari, Phys. Rev. B 80 (2009).

[30] H. Yoshida, Y. Kuwauchi, J.R. Jinschek, K.J. Sun, S. Tanaka, M. Kohyama, S. Shimada, M.

Haruta, S. Takeda, Science 335 (2012) 317.

[31] P. Pyykko, Angew. Chem. Int. Ed. 43 (2004) 4412.

[32] A. Ikeda, Y. Nakao, H. Sato, S. Sakaki, J. Phys. Chem. A 111 (2007) 7124.

[33] S. Grimme, J. Antony, S. Ehrlich, H. Krieg, J. Chem. Phys. 132 (2010) 154104.

[34] W.R. Tyson, W.A. Miller, Surf. Sci. 62 (1977) 267.

[35] K.J. Sun, M. Kohyama, S. Tanaka, S. Takeda, J. Phys. Chem. C 118 (2014) 1611.

[36] K.J. Sun, M. Kohyama, S. Tanaka, S. Takeda, ChemCatChem 5 (2013) 2217.

[37] J. Kim, E. Samano, B.E. Koel, Surf. Sci. 600 (2006) 4622.

[38] V.A. Bondzie, S.C. Parker, C.T. Campbell, J. Vac. Sci. Technol., A 17 (1999) 1717.

[39] H.Q. Shi, R. Asahi, C. Stampfl, Phys. Rev. B 75 (2007) 205125.

[40] N. Weiher, E.A. Willneff, C. Figulla-Kroschel, M. Jansen, S.L.M. Schroeder, Solid State Commun. 125 (2003) 317.

[41] B.W. Dodson, Phys. Rev. B 36 (1987) 6288.

[42] P.Y. Hsiao, Z.H. Tsai, J.H. Huang, G.P. Yu, Phys. Rev. B 79 (2009) 155414.

[43] S. Marchini, S. Gunther, J. Wintterlin, Phys. Rev. B 76 (2007).

[44] B. Wang, X.F. Ma, M. Caffio, R. Schaub, W.X. Li, Nano Lett. 11 (2011) 424.

[45] G. Wulff, Zeitschrift Fur Krystallographie Und Mineralogie 34 (1901) 449. 